\title{
Results on Almost Resilient Functions
}

\author{
Pinhui $\mathrm{Ke}^{1,3}$, Jie Zhang ${ }^{1,2}$, and Qiaoyan $\mathrm{Wen}^{1}$ \\ 1 School of Science, Beijing University of Posts and Telecommunications, \\ Beijing, 100876, P.R. China \\ keph@eyou.com \\ 2 State Key Laboratory of Information Security, \\ Chinese Academy of Sciences, Beijing 100039, P.R. China \\ 3 School of Mathematics and Computer Science, \\ Fujian Normal University, Fujian 350007, P.R. China
}

\begin{abstract}
Almost resilient function is the generalization of resilient function and has important applications in multiple authenticate codes and almost security cryptographic Boolean functions. In this paper, some constructions are provided. In particular, the Theorem 3 in [7] is improved. As $\varepsilon$-almost $(n, 1, k)$-resilient functions play an important role in the secondary constructions, we concluded some properties and constructions. Specially we presented a spectral characterization of almost $(n, 1, k)$-resilient functions, which can be used to identify an almost $(n, 1, k)$-resilient function by computing its walsh spectra.
\end{abstract}

Keywords: Almost resilient Function, Resilient function, Almost correlation immune function.

\section{Introduction}

A Boolean functions is a map from $F_{2}^{n}$ to $F_{2}$ and by a multi-output Boolean functions we mean a map from $F_{2}^{n}$ to $F_{2}^{m}$. They are used as basic primitives for designing ciphers. In order to resist known attacks, several criteria of Boolean functions have been developed. However there are some tradeoffs between these criteria. Strict fulfillment in one criterion may lead to weaken another one. For example, bent functions have the best nonlinearity, but they are never balanced or correlation immune. So we may relax the definition's conditions and functions with better parameters can be obtained.

The concept of a resilient function was first introduced by Chor et al. 1], which have been found to be applicable in fault-tolerant distribute computing, quantum cryptographic key distribution and so on. K.Kurosawa et al. 22] generalized the concept and introduced the definition of almost resilient function. An $\varepsilon$-almost $(n, m, k)$-resilient function is an $n$-input $m$-output function $f$ with the property that the deviation of output's distribution from uniform distribution is not great than $\varepsilon$ when $k$ arbitrary inputs are fixed and the remaining $n-k$ inputs run through all the $2^{n-k}$ input tuples. It was showed to have parameters superior to resilient function. As pointed out in [4, one important task of 
construction of vector resilient functions is to construct $(n, m, k)$-resilient functions with degree $d>m$ and high nonlinearity. It have been shown that we are able to improve the degree of the constructed functions with a small trade-off in the nonlinearity and resiliency. The notations of independent sample space was introduced by Naor and Naor [3], which had been proved to have many cryptographic applications, such as multiple authentication codes [5], almost security cryptographic Boolean functions [6] and so on. In [2], the relations between the almost resilient functions and the large sets of almost independent sample spaces were established. Recently, the relation between almost resilient function and its component functions was investigated in [7. They proved that if each nonzero linear combination of $f_{1}, f_{2}, \cdots, f_{m}$ is an $\varepsilon$-almost $(n, 1, k)$-resilient function, then $F=\left(f_{1}, f_{2}, \cdots, f_{m}\right)$ is a $\frac{2^{m}-1}{2^{m-1}} \varepsilon$-almost $(n, m, k)$-resilient function. However up to the present, the known constructions of almost resilient functions are by using almost independent sample space [2] and a constructions of almost $(3 n, 2,2 k+1)$ resilient function based on a balanced resilient function[7]. In Section 3, more constructions will be presented.

Because the close relation between almost resilient function and large set of almost independent sample space, the constructions of balanced almost resilient function are concerned. Balanced almost CI function plays an important role in the secondary construction of balanced almost resilient function. On the other hand by Siegenthalar's inequality, correlation immune order and algebraic degree are shown to be two contradictory criteria. Almost CI function was then proposed to solve this problem. Walsh Spectrum is useful tool in the characterization of CI function, which is known as Xiao-Massey theorem. But in the almost case, there is not such an efficient characterization. In Section 4, we will investigate this problem.

This paper is organized as follows. Some definitions and preliminaries that will be used later in the paper are described in Section 2. In Section 3, more constructions of almost resilient functions are provided. In section 4 , we conclude some construction methods and properties of almost $(n, 1, k)$-resilient functions. Especially we present a spectral characterization of almost $(n, 1, k)$-resilient functions and prove it is feasible to determine an almost $(n, 1, k)$-resilient function by computing its walsh spectra, which can be regarded as the generalization of Xiao-Massey theorem in the almost case to a certain extent.

\section{Preliminaries}

The vector spaces of $n$-tuples of elements from $\mathrm{GF}(2)$ is denoted by $F_{2}^{n}$. Let $F$ be a function from $F_{2}^{n}$ to $F_{2}^{m}$.

Definition 1. The function $F$ is called an $(n, m, k)$-resilient function if

$$
\operatorname{Pr}\left[F\left(x_{1}, \cdots, x_{n}\right)=\left(y_{1}, \cdots, y_{m}\right) \mid x_{i_{1}} x_{i_{2}} \cdots_{i_{k}}=\alpha\right]=2^{-m}
$$

for any $k$ positions $i_{1}<i_{2}<\cdots<i_{k}$, for any $k$-bit string $\alpha \in F_{2}^{k}$, and for any $\left(y_{1}, \cdots, y_{m}\right) \in F_{2}^{m}$, where the values $x_{j}\left(j \notin\left\{i_{1}, i_{2}, \cdots, i_{k}\right\}\right)$ are chosen independently at random. 
Following propositions are well-known and useful in understanding the relationship between a resilient functions and its component functions. It has appeared in many references (see, for example, [9]).

Proposition 1. Let $F=\left(f_{1}, \cdots, f_{m}\right)$ be a function from $F_{2}^{n}$ to $F_{2}^{m}$, where $n$ and $m$ are integers with $n \geq m \geq 1$, and each $f_{i}$ is a function on $F_{2}^{n}$. Then $F$ is an $(n, m, k)$-resilient function if and only if every nonzero combination of $f_{1}, \cdots, f_{m}$

$$
f(x)=\bigoplus_{i=1}^{m} c_{i} f_{i}(x)
$$

is a $(n, 1, k)$-resilient function, where $c=\left(c_{1}, \cdots, c_{n}\right) \in F_{2}^{n}$.

K.Kurosawa et al. introduced a notation of $\varepsilon$-almost $(n, m, k)$-resilient function [2].

Definition 2. The function $F$ is called a $\varepsilon$-almost $(n, m, k)$-resilient function if

$$
\left|\operatorname{Pr}\left[F\left(x_{1}, \cdots, x_{n}\right)=\left(y_{1}, \cdots, y_{m}\right) \mid x_{i_{1}} x_{i_{2}} \cdots_{i_{k}}=\alpha\right]-2^{-m}\right| \leq \varepsilon
$$

for any $k$ positions $i_{1}<i_{2}<\cdots<i_{k}$, for any $k$-bit string $\alpha \in F_{2}^{k}$, and for any $\left(y_{1}, \cdots, y_{m}\right) \in F_{2}^{m}$, where the values $x_{j}\left(j \notin\left\{i_{1}, i_{2}, \cdots, i_{k}\right\}\right)$ are chosen independently at random.

By the definition, it is easy to prove following lemma.

Lemma 1. If $F$ is an $\varepsilon$-almost $(n, m, k)$-resilient function, then $F$ is also an $\varepsilon$-almost $(n, m, r)$-resilient function for any $r \leq k$.

An almost $k$-wise independent sample space is probability space on $n$-bit tuples such that any $k$-bits are almost independent. A large set of $(\varepsilon, k)$-independent sample spaces, denoted by $L S(\varepsilon, k, n, t)$, is a set of $2^{m-t}(\varepsilon, k)$-independent sample spaces, each of size $2^{t}$, such their union contains all $2^{n}$ binary vectors of length $n$. For details about $k$-wise independent sample spaces and $L S(\varepsilon, k, n, t)$, we refer to 2,3$]$.

The relation between $L S(\varepsilon, k, n, t)$ and almost resilient function is revealed in 2 .

Proposition 2. If there exists an $L S(\varepsilon, k, n, t)$, then there exists a $\delta$-almost $(n, n-t, k)$-resilient function, where $\delta=\frac{\varepsilon}{2^{n-t-k}}$.

A $(n, m)$-function $F$ is called balanced if

$$
\operatorname{Pr}\left[F\left(x_{1}, \cdots, x_{n}\right)=\left(y_{1}, \cdots, y_{m}\right)\right]=2^{-m}
$$

for all $\left(y_{1}, \cdots, y_{m}\right) \in F_{2}^{m}$.

Proposition 3. If there exists a balanced $\varepsilon$-almost $(n, m, k)$-resilient function, then there exists a $L S(\delta, k, n, n-m)$, where $\delta=\frac{\varepsilon}{2^{k-m}}$. 
Using Weil-Carlitz-Uchiyama bound, K.Kurosawa et al. 2] present a construction of $t$-systematic $(\varepsilon, k)$-independent sample spaces and then extended to large set of almost independent sample spaces. So by Proposition 2, some almost resilient functions are obtained.

Let $F(X)=\left(f_{1}, f_{2}, \cdots, f_{m}\right)$ be an $(n, m)$-function, the nonlinearity of $F$ is defined to be $n l(F)=\min \{n l(l \circ f): l$ is a non-constant $m$-variable linear function\}, where $n l(f)$ is the least hamming distance between Boolean function $f$ and all affine functions. And the degree of $F$ defined to be the minimum of the degree of $l \circ f$, where $l$ ranges over all non-constant $m$-variable linear function.

Similar to the resilient function, correlation immune function can also be generalized. K. Kurosawa et al. 2] called it the almost correlation immune function. In fact, an earlier generalization version of the single output case has been introduced by Yixian Yan [1].

Definition 3. The function $F$ is called an $\varepsilon$-almost $(n, m, k)$-correlation immune function if

$$
\begin{gathered}
\mid \operatorname{Pr}\left[F\left(x_{1}, \cdots, x_{n}\right)=\left(y_{1}, \cdots, y_{m}\right) \mid x_{i_{1}} x_{i_{2}} \cdots_{i_{k}}=\alpha\right]-\operatorname{Pr}\left[F\left(x_{1}, \cdots, x_{n}\right)=\left(y_{1},\right.\right. \\
\left.\left.\cdots, y_{m}\right)\right] \mid \leq \varepsilon
\end{gathered}
$$

for any $k$ positions $i_{1}<i_{2}<\cdots<i_{k}$, for any $k$-bit string $\alpha \in F_{2}^{k}$, and for any $\left(y_{1}, \cdots, y_{m}\right) \in F_{2}^{m}$, where the values $x_{j}\left(j \notin\left\{i_{1}, i_{2}, \cdots, i_{k}\right\}\right)$ are chosen independently at random.

The relation between almost CI function and nonuniform $L S(\varepsilon, k, n, t)$ is given in [2]. It is easy to see that an $\varepsilon$-almost $(n, m, k)$-resilient function is equivalent to an balanced $\varepsilon$-almost $(n, m, k)$-CI function.

Let $f$ be a function from $F_{2}^{n}$ to $F_{2}$, then

$$
S_{f}(w)=\sum_{x \in F_{2}^{n}}(-1)^{f(x) \oplus w \cdot x}
$$

is called a Walsh transformation of $f$. Walsh transform is a useful tool and many cryptographic criteria of a Boolean function can be characterized by it.

\section{Construction of Almost Resilient Functions}

In the following, if $h$ is a functions from $F_{2}^{n}$ to $F_{2}^{m}$ or $F_{2}$, denote

$$
L(h(X)=Y)=\left\{\left(x_{1}, x_{2}, \cdots, x_{n}\right): h\left(x_{1}, x_{2}, \cdots, x_{n}\right)=Y\right\} .
$$

Let $X_{i}, 1 \leq i \leq m$, be $m$ independent random variables on $F_{2}$. The number of nonzero combination of $X_{1}, X_{2}, \cdots, X_{m}$ is $C_{m}^{1}+C_{m}^{2}+\cdots+C_{m}^{m}=2^{m}-1$. We divide it into two parts, each contains $2^{m-1}$ and $2^{m-1}-1$ elements respectively. Denote them as $A_{1}$ and $A_{2}$. For a fixed $\left(y_{1}, y_{2}, \cdots, y_{m}\right) \in F_{2}^{m}$ and a nonzero linear combination of $X_{1}, X_{2}, \cdots, X_{m}$, it determine a set $L\left(\oplus_{i=1}^{m} c_{i} X_{i}=\right.$ 
$\left.\oplus_{i=1}^{m} c_{i} y_{i}\right)$. We call the set determined by $\left(y_{1}, y_{2}, \cdots, y_{m}\right)$. Furthermore we call the set $L\left(\oplus_{i=1}^{m} c_{i} X_{i}=\oplus_{i=1}^{m} c_{i} y_{i} \oplus 1\right)$ the determined complement set induced by $\left(y_{1}, y_{2}, \cdots, y_{m}\right)$. For each nonzero $m$-bit string $\left(c_{1}, c_{2}, \cdots, c_{m}\right) \in F_{2}^{m}$ and $a \in F_{2}$, by (1) it is obvious that

$$
\left|L\left(\oplus_{i=1}^{m} c_{i} X_{i}=a\right)\right|=2^{m-1}, L\left(\oplus_{i=1}^{m} c_{i} X_{i}=0\right) \cup L\left(\oplus_{i=1}^{m} c_{i} X_{i}=1\right)=F_{2}^{m} .
$$

Lemma 2. 77 Let notations defined as above. For an arbitrary $m$-bit string $Y=\left(y_{1}, y_{2}, \cdots, y_{m}\right) \in F_{2}^{m}$, then the collection of determined sets of $A_{1}$ equals to the collection of determined complement sets of $A_{2}$ added $2^{m-1} Y$. Note again that we call the two collections are equal if and only if the elements and its multiplicity in the two collections are identical.

In [7, relations between almost resilient function and its component functions were presented.

Theorem 1. Let $F=\left(f_{1}, \cdots, f_{m}\right)$ be a function from $F_{2}^{n}$ to $F_{2}^{m}$, where $n$ and $m$ are integers with $n \geq m \geq 1$, and each $f_{i}$ is a function on $F_{2}^{n}$. If $F$ is an $\varepsilon$-almost $(n, m, k)$-resilient function, then each nonzero combination of $f_{1}, \cdots, f_{m}$

$$
f(x)=\bigoplus_{i=1}^{m} c_{i} f_{i}(x)
$$

is a $2^{m-1} \varepsilon$-almost $(n, 1, k)$-resilient function.

Theorem 2. Let $F=\left(f_{1}, \cdots, f_{m}\right)$ be a function from $F_{2}^{n}$ to $F_{2}^{m}$, where $n$ and $m$ are integers with $n \geq m \geq 1$, and each $f_{i}$ is a function on $F_{2}^{n}$. If each nonzero combination of $f_{1}, \cdots, f_{m}$

$$
f(x)=\bigoplus_{i=1}^{m} c_{i} f_{i}(x)
$$

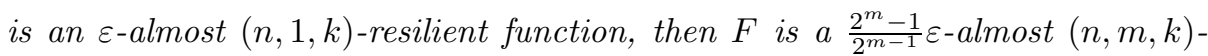
resilient function, where $x=\left(x_{1}, \cdots, x_{n}\right) \in F_{2}^{n}$.

Remark. By Theorem 1 for any $\varepsilon$-almost $(n, m, k)$-resilient function $F=\left(f_{1}, \ldots, f_{m}\right)$ every nonzero linear combination of $f_{i}$ is an $2^{m-1} \varepsilon$-almost $(n, 1, k)$-resilient function. But by Theorem 2 if every nonzero linear combination is an $2^{m-1} \varepsilon$-almost $(n, 1, k)$-resilient function then $\left(f_{1}, \ldots, f_{m}\right)$ is $\left(2^{m}-1\right) \varepsilon$-almost $(n, m, k)$-resilient function. Thus starting from $\varepsilon$-almost $(n, m, k)$-resilient function one can obtain an $\left(2^{m}-1\right) \varepsilon$-almost $(n, m, k)$-resilient function. This gap between $\varepsilon$ and $\left(2^{m}-1\right) \varepsilon$ implies that both statements are not equally strong. Compared with Proposition 1 we could see although the almost resilient function only bias $\varepsilon$ from resilient function in definition it is difficult for us to prove the same proposition of resilient function in almost case.

A construction based on a balanced almost $(n, 1, k)$-resilient function was presented in [7]. 
Theorem 3. Let $f$ be a balanced $\varepsilon$-almost $(n, 1, k)$-resilient function, then

$$
g(X, Y, Z)=(f(X) \oplus f(Y), f(Y) \oplus f(Z))
$$

is a balanced $\frac{9}{2} \varepsilon$-almost $(3 n, 2,2 k+1)$-resilient function.

But the proof of the theorem is tedious. Here we present a direct proof and improve the result.

Theorem 4. Let $f$ be a balanced $\varepsilon$-almost $(n, 1, k)$-resilient function, then

$$
g(X, Y, Z)=(f(X) \oplus f(Y), f(Y) \oplus f(Z))
$$

is a balanced $\frac{3}{2} \varepsilon$-almost $(3 n, 2,2 k+1)$-resilient function.

Proof. Denote $h(X, Y)=f(X) \oplus f(Y)$. It is obvious that $h(X, Y)$ is balanced. We first prove that

$$
\left|\operatorname{Pr}\left(h(X, Y)=1 \mid x_{i_{1}} \cdots x_{i_{r}} y_{i_{r+1}} \cdots y_{i_{2 k+1}}\right)-\frac{1}{2}\right| \leq \varepsilon
$$

for any $2 k+1$ positions $x_{i}, 1 \leq i \leq r$ and $y_{j}, r+1 \leq j \leq 2 k+1$.

Without loss of generality, we may assume $r \leq k$. Then by Lemma 1 , for any $a \in F_{2}$,

$$
\left|\operatorname{Pr}\left(f(X)=a \mid x_{i_{1}} \cdots x_{i_{r}}\right)-\frac{1}{2}\right| \leq \varepsilon .
$$

By notation (1), for any $a \in F_{2}$, we have

$$
2^{n-r-1}-2^{n-r} \varepsilon \leq\left|L\left(f(X)=a \mid x_{i_{1}} \cdots x_{i_{r}}\right)\right| \leq 2^{n-r-1}+2^{n-r} \varepsilon .
$$

Because we are taking a direct sum of $f(X)$ and $f(Y)$, therefore we can deduce the bounds for the $\left|L\left(f(X) \oplus f(Y)=1 \mid x_{i_{1}} \cdots x_{i_{r}} y_{i_{r+1}} \cdots y_{i_{2 k+1}}\right)\right|$ by multiplying the previous inequality for $\left|L\left(f(X)=a \mid x_{i_{1}} \cdots x_{i_{r}}\right)\right|$ with the weight of $\left\{f(Y) \mid y_{i_{r+1}} \cdots y_{i_{2 k+1}}\right\}$. That is

$$
\begin{gathered}
2^{n-(2 k+1-r)}\left(2^{n-r-1}-2^{n-r} \varepsilon\right) \leq\left|L\left(f(X) \oplus f(Y)=1 \mid x_{i_{1}} \cdots x_{i_{r}} y_{i_{r+1}} \cdots y_{i_{2 k+1}}\right)\right| \\
\leq 2^{n-(2 k+1-r)}\left(2^{n-r-1}+2^{n-r} \varepsilon\right) .
\end{gathered}
$$

i.e.

$$
\left|\operatorname{Pr}\left(h(X, Y)=1 \mid x_{i_{1}} \cdots x_{i_{r}} y_{i_{r+1}} \cdots y_{i_{2 k+1}}\right)-\frac{1}{2}\right| \leq \varepsilon .
$$

So $h(X, Y)$ is a balanced $\varepsilon$-almost $(2 n, 1,2 k+1)$ resilient function. The case $f(Y) \oplus f(Z)$ and $f(X) \oplus f(Z)$ can be similarly proved. And each of them is balanced, so $g$ is also balanced. By Theorem 2, the proof is completed.

We can generalize above result as follows.

Theorem 5. Let $f_{i}$ be a balanced $\varepsilon_{i}$-almost $\left(n_{i}, 1, k_{i}\right)$-resilient function, $1 \leq i \leq$ $l, G$ be a $[l, m, d]$ linear code. Then

$$
F\left(X_{1}, X_{2}, \cdots, X_{l}\right)=\left(f_{1}\left(X_{1}\right), f_{2}\left(X_{2}\right), \cdots, f_{l}\left(X_{l}\right)\right) G^{T}
$$

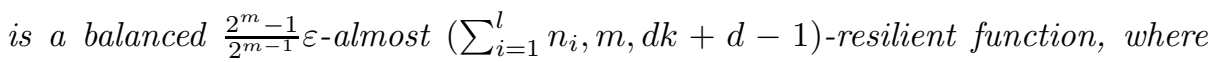
$k=\min _{1 \leq i \leq l} k_{i}$ and $\varepsilon=\max _{1 \leq i \leq l} \varepsilon_{i}$. 
Proof. Assume that $G=\left[a_{i j}\right], 1 \leq i \leq m, 1 \leq j \leq l$. Then

$$
F=\left(\oplus_{i=1}^{l} a_{1 i} f_{i}, \oplus_{i=1}^{l} a_{2 i} f_{i}, \cdots, \oplus_{i=1}^{l} a_{m i} f_{i}\right) .
$$

For any nonzero linear combination of its component functions of $F$,we have

$$
\oplus_{j=1}^{m} c_{j}\left(\oplus_{i=1}^{l} a_{j i} f_{i}\right)=\oplus_{i=1}^{l} f_{i}\left(\oplus_{j=1}^{m} c_{j} a_{j i}\right)
$$

where $c=\left(c_{1}, \cdots, c_{m}\right) \in F_{2}^{m}$ is a nonzero vector. And note that minimum weight of code of $G$ is $d$. So at least $d$ functions of $f_{1}, \cdots, f_{l}$ appear in above formulation. Similar to the proof in Theorem 4, we known that any nonzero linear combination of its component functions of $F$ is $\varepsilon$-almost $\left(\sum_{i=1}^{l} n_{i}, 1, d k+d-1\right)$ resilient function. By Theorem 2, we complete the proof.

Corollary 1. If there exit an $[l, m, d]$ linear code and $\varepsilon$-almost $(n, 1, k)$-resilient function, then an $\frac{2^{m}-1}{2^{m-1}} \varepsilon$-almost $(l n, m, d k+d-1)$-resilient function must exist.

If we take $f_{1}=f_{2}=f_{3}=f$ and

$$
G=\left[\begin{array}{lll}
1 & 1 & 0 \\
0 & 1 & 1
\end{array}\right]
$$

then Theorem 4 may be regarded as a corollary of Theorem 5 .

Theorem 6. Let $F=\left(f_{1}, \cdots, f_{m}\right)$ be $\varepsilon_{1}$-almost $\left(n_{1}, m, t_{1}\right)$-resilient function and $G=\left(g_{1}, \cdots, g_{m}\right)$ be $\varepsilon_{2}$-almost $\left(n_{2}, m, t_{2}\right)$-resilient function.Then $F(X) \oplus$ $G(Y)=\left(f_{1}(x) \oplus g_{1}(y), \cdots, f_{m}(x) \oplus g_{m}(y)\right)$ is $\varepsilon$-almost $\left(n_{1}+n_{2}, m, t_{1}+t_{2}+1\right)$ resilient function, where $\varepsilon=\max \left(\varepsilon_{1}, \varepsilon_{2}\right)$.

Proof. By definition, we need to prove that

$$
\left|\operatorname{Pr}\left(F(X) \oplus G(Y)=\eta \mid x_{i_{1}} \cdots x_{i_{r}} y_{i_{r+1}} \cdots y_{i_{t_{1}+t_{2}+1}}\right)-\frac{1}{2^{m}}\right| \leq \varepsilon
$$

holds for arbitrary chosen $\eta \in F_{2}^{m}$ and for any $t_{1}+t_{2}+1$ positions $x_{i}, 1 \leq i \leq r$ and $y_{i}, r+1 \leq i \leq t_{1}+t_{2}+1$.

Without loss of generality, assume that $r \leq t_{1}$. Then for arbitrary $G(Y)=\alpha$, there exist exactly one $\beta \in F_{2}^{m}$ such that $F(X)=\beta$ and $F(X)+\alpha=\eta$. For $r \leq t_{1}$, we have

$$
\left|\operatorname{Pr}\left(F(X)=\beta \mid x_{i_{1}} \cdots x_{i_{r}}\right)-\frac{1}{2^{m}}\right| \leq \varepsilon
$$

i.e.

$$
2^{n_{1}-r-m}-2^{n_{1}-r} \varepsilon \leq\left|L\left(F(X)=\beta \mid x_{i_{1}} \cdots x_{i_{r}}\right)\right| \leq 2^{n_{1}-r-m}+2^{n_{1}-r} \varepsilon .
$$

So we have

$$
\begin{gathered}
2^{n_{2}-\left(t_{1}+t_{2}+1-r\right)}\left(2^{n_{1}-r-m}-2^{n_{1}-r} \varepsilon\right) \leq \mid L\left(F(X) \oplus G(Y)=\eta \mid x_{i_{1}} \cdots x_{i_{r}}\right. \\
\left.y_{i_{r+1}} \cdots y_{i_{t_{1}+t_{2}+1}}\right) \mid \leq 2^{n_{2}-\left(t_{1}+t_{2}+1-r\right)}\left(2^{n_{1}-r-m}+2^{n_{1}-r} \varepsilon\right) .
\end{gathered}
$$


That is

$$
\begin{gathered}
2^{n_{1}+n_{2}-\left(t_{1}+t_{2}+1\right)-m}-2^{n_{1}+n_{2}-\left(t_{1}+t_{2}+1\right)} \varepsilon \leq \mid L\left(F(X) \oplus G(Y)=\eta \mid x_{i_{1}} \cdots x_{i_{r}}\right. \\
\left.y_{i_{r+1}} \cdots y_{i_{t_{1}+t_{2}+1}}\right) \mid \leq 2^{n_{1}+n_{2}-\left(t_{1}+t_{2}+1\right)-m}+2^{n_{1}+n_{2}-\left(t_{1}+t_{2}+1\right)} \varepsilon .
\end{gathered}
$$

Thus we know that $F \oplus G$ is $\varepsilon$-almost $\left(n_{1}+n_{2}, m, t_{1}+t_{2}+1\right)$-resilient function.

The conclusion of Theorem 6 can be slightly generalized with a similar proof.

Theorem 7. Let $F_{i}(X), 1 \leq i \leq l$, be $\varepsilon_{i}$-almost $\left(n_{i}, m, t_{i}\right)$-resilient function. Then $\oplus F_{i}\left(X_{i}\right)$ is an $\varepsilon$-almost $\left(\sum_{i=1}^{l} n_{i}, m, \sum_{i=1}^{l} t_{i}+l-1\right)$ resilient function, where $\varepsilon=\max _{1 \leq i \leq l} \varepsilon_{i}$.

The Theorem 4.1 in [8] could be generalized to almost case.

Theorem 8. Let $F(X)$ be an $\varepsilon$-almost $(n, m, t)$-resilient function and $G$ be a $[N, k, d]$ linear code.Then

$$
H\left(X_{1}, X_{2}, \cdots, X_{N}\right)=\left(F\left(X_{1}\right), F\left(X_{2}\right), \cdots, F\left(X_{N}\right)\right) G^{T}
$$

is an $\frac{2^{k m}-1}{2^{k m}-m} \varepsilon$-almost $(n N, m k, d(t+1)-1)$-resilient function.

Proof. The proof is similar to that of $[8$. The only difference is that any nonzero linear combination of component functions of $F(X)$ is an $2^{m-1} \varepsilon$-almost $(n, 1, t)$ -resilient function by Theorem 1. By Theorem 2 again, the proof is completed.

Just as we point out in the above remark, the gap between Theorem 1 and 2 is responsible for the increasing of $\varepsilon$ in former secondary constructions. So although Theorem 5 may be seemed as a special case of Theorem 8 (let $\mathrm{m}=1$ ), we would prefer to Theorem 5 in secondary construction of almost resilient function under the present condition.

\section{Spectral Characterization of $\varepsilon$-Almost $(n, 1, k)$ - Resilient Functions}

As we have described in the last paragraph of Section 3 and Proposition 3, we are interest in balanced $\varepsilon$-almost $(n, 1, k)$-CI functions , i.e. $\varepsilon$-almost $(n, 1, k)$ resilient functions. Furthermore as we have known that the algebraic degree and correlation immune order is incompatible, almost CI function is also proposed to avoid this dilemma when it was used as combination or filter function in stream cipher.

Some constructions of almost CI functions had been presented in [1].

Theorem 9. 11] Let $f$ be a $k$ order CI function and $g$ be a functions such that $w t(g)$ is a little number. Then $h=f \oplus g$ is a $\frac{3+2^{k+1}}{2^{n}} w t(g)$-almost $(n, 1, k)-C I$ function.

Theorem 10. [11] Let $f_{1}$ be a balanced $\varepsilon_{1}$-almost $(n, 1, k)$-CI function and $f_{2}$ be a balanced $\varepsilon_{2}$-almost $(n, 1, k)$-CI function. Then $f\left(x_{1}, \cdots, x_{n}, x_{n+1}\right)=$ $x_{n+1} f_{1} \oplus\left(1 \oplus x_{n+1}\right) f_{2}$ is a balanced $\varepsilon$-almost $(n+1,1, k)$-CI function, where $\varepsilon=\max \left(\varepsilon_{1}, \varepsilon_{2}\right)$. 
We could see that it is easy to obtain an almost balanced CI function by modifying a CI function slightly. In this way we may derive many constructions.

It is well known that $f$ is a $(n, 1, k)$-CI function if and only if each $f \bigoplus \oplus_{i=1}^{n} a_{i} x_{i}$ is a balanced function for all $1 \leq w t(\alpha) \leq k, \alpha=\left(a_{1}, a_{2}, \cdots, a_{n}\right) \in F_{2}^{n}$. It can be restated in the word of walsh transform, which is the well-known Xiao-Massey theorem. In the almost case, Yixian Yan [1] presented the following result.

Theorem 11. Let $f$ be an $\varepsilon$-almost $(n, 1, k)$-CI function, then

$$
\left|\operatorname{Pr}\left(f(X) \bigoplus \oplus_{i=1}^{n} a_{i} x_{i}=1\right)-\frac{1}{2}\right| \leq \varepsilon
$$

for any $1 \leq w t(\alpha) \leq k, \alpha=\left(a_{1}, a_{2}, \cdots, a_{n}\right) \in F_{2}^{n}$.

It means that for an $\varepsilon$-almost $(n, 1, k)$-CI function the function $f \bigoplus \oplus_{i=1}^{n} a_{i} x_{i}$ should be almost balanced for all $\alpha, 1 \leq w t(\alpha) \leq k$. Now Let us consider the opposite direction, i.e. if a function $f$ such that $f \bigoplus \oplus_{i=1}^{n} a_{i} x_{i}$ is almost balanced for all $\alpha, 1 \leq w t(\alpha) \leq k$, is the function $f$ an almost $(n, 1, k)$-CI function? It is an interesting problem because if it holds we will be able to identify an almost CI function by computing its walsh spectra. Firstly in the case $k=1$ and $f$ is balanced, we have the following lemma.

Lemma 3. Let $f$ be a function from $F_{2}^{n}$ to $F_{2}$. If $f$ is balanced and

$$
\left|\operatorname{Pr}\left(f(X) \oplus x_{i}=1\right)-\frac{1}{2}\right| \leq \varepsilon
$$

holds for any $1 \leq i \leq n$ if and only if $f$ is an $\varepsilon$-almost $(n, 1,1)$-CI function.

Proof. By Theorem 11, the sufficiency is obvious. Let us prove the necessity. Without lost of generality, we assume $i=1$. Denote $P_{i j}=\operatorname{Pr}\left(f(X)=i \mid x_{1}=\right.$ $j), 0 \leq i, j \leq 1$. It is easy to verify that

$$
\begin{array}{r}
P_{00}+P_{01}=2 \operatorname{Pr}(f(x)=0), P_{10}+P_{11}=2 \operatorname{Pr}(f(x)=1), \\
P_{00}+P_{10}=P_{01}+P_{11}=1 .
\end{array}
$$

By the condition of the lemma, we have

$$
\left|\operatorname{Pr}\left(f(X) \oplus x_{1}=1\right)-\frac{1}{2}\right| \leq \varepsilon .
$$

Furthermore,

$$
\operatorname{Pr}\left(f(X) \oplus x_{1}=1\right)=\operatorname{Pr}\left(f(X)=1, x_{1}=0\right)+\operatorname{Pr}\left(f(X)=0, x_{1}=1\right)=\frac{1}{2}\left(P_{10}+P_{01}\right) .
$$

Hence,

$$
1-2 \varepsilon \leq P_{10}+P_{01} \leq 1+2 \varepsilon .
$$

From (3) and (5), we have

$$
\begin{gathered}
1-2 \varepsilon+2 \operatorname{Pr}(f(X)=1) \leq P_{10}+P_{01}+P_{10}+P_{11} \leq 1+2 \varepsilon+2 \operatorname{Pr}(f(X)=1), \\
1-2 \varepsilon+2 \operatorname{Pr}(f(X)=1) \leq 2 P_{10}+P_{01}+P_{11} \leq 1+2 \varepsilon+2 \operatorname{Pr}(f(X)=1) .
\end{gathered}
$$


By (4),

$$
-\varepsilon \leq P_{10}-\operatorname{Pr}(f(X)=1) \leq \varepsilon
$$

Thus,

$$
\left|\operatorname{Pr}\left(f(X)=1 \mid x_{1}=0\right)-\operatorname{Pr}(f(x)=1)\right| \leq \varepsilon .
$$

Similarly we can prove

$$
\left|\operatorname{Pr}\left(f(X)=1 \mid x_{i}=a\right)-\operatorname{Pr}(f(x)=1)\right| \leq \varepsilon, \text { for any } 1 \leq i \leq n, a \in F_{2} .
$$

For $f$ is balanced,

$$
\left|\operatorname{Pr}\left(f(X)=1 \mid x_{i}=a\right)-\frac{1}{2}\right| \leq \varepsilon .
$$

Thus the proof is completed.

Now we prove the main result.

Theorem 12. Let $f$ be a function from $F_{2}^{n}$ to $F_{2}$. If $f$ is balanced and

$$
\left|\operatorname{Pr}\left(f(X) \bigoplus \oplus c_{i} x_{i}=1\right)-\frac{1}{2}\right| \leq \varepsilon
$$

holds for any $c=\left(c_{1}, c_{2}, \cdots, c_{n}\right) \in F_{2}^{n}$ and $1 \leq w t(c) \leq k$. Then $f$ is an $\left(2^{k}-1\right) \varepsilon$-almost $(n, 1, k)$-resilient function.

Proof. We prove the theorem in three steps.

1. For a fixed nonzero vector $c \in F_{2}^{n}$,

$$
\left|\operatorname{Pr}\left(f(X) \bigoplus \oplus c_{i} x_{i}=1\right)-\frac{1}{2}\right| \leq \varepsilon,
$$

then we have

$$
\left|\operatorname{Pr}\left(\oplus c_{i} x_{i}=1 \mid f(X)\right)-\frac{1}{2}\right| \leq \varepsilon .
$$

Note that $c \neq(0,0, \cdots, 0)$ and $f$ is balanced. The proof of step 1 is similar to that of Lemma 3 .

2. If

$$
\left|\operatorname{Pr}\left(\oplus_{i=1}^{k} c_{i} x_{i} \mid f(X)\right)-\frac{1}{2}\right| \leq \varepsilon,
$$

then we have

$$
\left|\operatorname{Pr}\left(x_{1} \cdots x_{k} \mid f(X)\right)-\frac{1}{2^{k}}\right| \leq \frac{2^{k}-1}{2^{k-1}} \varepsilon .
$$

Divide all the nonzero linear combinations of $x_{1}, \cdots, x_{k}$ into two part $A_{1}$ and $A_{2}$, such that $\left|A_{1}\right|=2^{k-1}$ and $\left|A_{2}\right|=2^{k-1}-1$. For any fixed $\alpha=\left(a_{1}, \cdots, a_{k}\right) \in$ $F_{2}^{k}$, by Lemma 2 , we have

$$
\begin{gathered}
\sum_{c \in A_{1}}\left|L\left(\oplus c_{i} x_{i}=c_{i} a_{i} \mid f(X)\right)\right|=\sum_{c^{\prime} \in A_{2}}\left|L\left(\oplus c_{i}^{\prime} x_{i}=c_{i}^{\prime} a_{i} \oplus 1 \mid f(X)\right)\right| \\
+2^{k-1}\left|L\left(\left(x_{1}, \cdots, x_{k}\right)=\left(a_{1}, \cdots, a_{k}\right) \mid f(X)\right)\right| .
\end{gathered}
$$


By the condition of theorem and step 1, we know (7) holds for any nonzero vector $c=\left(c_{1}, c_{2}, \cdots, c_{n}\right) \in F_{2}^{n}$ and $1 \leq w t(c) \leq k$. So $(7)$ holds for any nonzero vector $c \in F_{2}^{k}$.

By

$$
\begin{gathered}
2^{k-1}\left(\frac{1}{2}-\varepsilon\right) \leq \sum_{c \in A_{1}} \operatorname{Pr}\left(\oplus c_{i} x_{i} \mid f(X)\right) \leq 2^{k-1}\left(\frac{1}{2}+\varepsilon\right), \\
\left(2^{k-1}-1\right)\left(\frac{1}{2}-\varepsilon\right) \leq \sum_{c^{\prime} \in A_{2}} \operatorname{Pr}\left(\oplus c_{i}^{\prime} x_{i} \mid f(X)\right) \leq\left(2^{k-1}-1\right)\left(\frac{1}{2}+\varepsilon\right),
\end{gathered}
$$

and (9), we have

$$
\frac{1}{2}-\left(2^{k}-1\right) \varepsilon \leq 2^{k-1} \operatorname{Pr}\left(\left(x_{1}, \cdots, x_{k}\right)=\left(a_{1}, \cdots, a_{k}\right) \mid f(X)\right) \leq \frac{1}{2}+\left(2^{k}-1\right) \varepsilon .
$$

That is

$$
\left|\operatorname{Pr}\left(\left(x_{1}, \cdots, x_{k}\right)=\left(a_{1}, \cdots, a_{k}\right) \mid f(X)\right)-\frac{1}{2^{k}}\right| \leq \frac{2^{k}-1}{2^{k-1}} \varepsilon .
$$

3. It is easy to verified that

$$
\operatorname{Pr}\left(f(X) \mid x_{1} \cdots x_{k}\right)=2^{k-1} \operatorname{Pr}\left(x_{1} \cdots x_{k} \mid f(X)\right) .
$$

So by (8) we have

$$
\left|\operatorname{Pr}\left(f(X) \mid x_{1} \cdots x_{k}\right)-\frac{1}{2}\right|=2^{k-1}\left|\operatorname{Pr}\left(x_{1} \cdots x_{k} \mid f(X)\right)-\frac{1}{2^{k}}\right| \leq\left(2^{k}-1\right) \varepsilon .
$$

Thus we have done.

Corollary 2. Let $f$ be a function from $F_{2}^{n}$ to $F_{2}$. If $S_{f}(0)=0$ and $\left|S_{f}(w)\right| \leq$ $2^{n+1} \varepsilon$ for any $w \in F_{2}^{n}, 1 \leq w t(w) \leq k$, then $f$ is an $\left(2^{k}-1\right) \varepsilon$-almost $(n, 1, k)$ resilient function.

Proof. Note that $\left|\operatorname{Pr}\left(f(X) \oplus \oplus w_{i} x_{i}=1\right)-\frac{1}{2}\right| \leq \varepsilon$ holds if and only if $-2^{n} \varepsilon+$ $2^{n-1} \leq w t(f(X) \oplus w \cdot X) \leq 2^{n} \varepsilon+2^{n-1}$. And $f$ is balanced if and only if $S_{f}(0)=0$. By $S_{f}(w)=\sum_{x \in F_{2}^{n}}(-1)^{f(x) \oplus w \cdot x}=2^{n}-2 w t(f(x) \oplus w \cdot x)$ and Theorem 12, the result is obtained.

By Corollary 2, it is convenient for us to identify an almost $(n, 1, k)$-resilient function by computing its walsh spectra.

\section{Conclusion}

In this paper, some constructions of almost resilient function are presented. For the relation between almost resilient function and large set of almost independent sample space, the constructions of balanced almost resilient function are concerned. As almost $(n, 1, k)$-resilient function play an important role in the secondary construction, we conclude some constructions methods. Especially we prove it is feasible to determine weather a function is an almost $(n, 1, k)$-resilient function by computing its walsh spectra, which can be regard as the generalization of Xiao-Massey theorem in the almost case to a certain extent. 


\section{Acknowledgements}

The authors wish to thank the referees for their comments and suggestions that helped to improved the correspondence. This work was supported by the National Natural Science Foundation of China (No.60373059), the National Research Foundation for the Doctoral Program of Higher Education of China (No.20040013007) and the Major Research Plan of the National Natural Science Foundation of China(Grant No. 90604023).

\section{References}

1. B.Chor, O.Goldreich, J.Hästad, J.Friedman, S.Rudich, and R.Smoledsky. The bit extraction problem or t-resilient functions. IEEE Symp. on Foundations of Computer Science, 1985, Vol.26, pp.396-407.

2. K.Kurosawa, T.Johansson,D.Stinson. Almost k-wise independent sample spaces and their applications. J.Cryptology, 2001, Vol.14, no.4, pp.231-253.

3. J.Naor, M.Naor. Small bias probality spaces:efficient constructions and applications. SIAM Journal on Computing 1993, Vol.22, pp.838-856.

4. K.C.Gupta, P.Sarkar. Improved construction of nonlinear resilient S - Boxes. IEEE Tran. on Info. Theory, 2005, Jan., Vol.51, No.1, pp.339-348.

5. M.N.Wegman, J.L.Carter. New hash functions and their use in authentication and set equality. Journal of Computer and System Sciences ,1981, Vol.22, PP.265-279.

6. K.Kurosawa, R.Matsumoto. Almost security of cryptographic Boolean functions,IEEE Tran. on Info. Theory, 2004, Vol.50, No.11, PP.2752-2761.

7. Pinhui Ke, Tailin Liu, Qiaoyan Wen. Construction of almost resilient functions. Cryptology and Network Security: 4th International Conference, CANS 2005,Yvo G. Desmedt et al. ed. LNCS 3810, Springer-Verlag 2005, pp.236-246.

8. Chuankun $\mathrm{Wu}, \mathrm{Ed}$ Dawson. On construction of resilient functions. Information Security and Privacy,Proceedings of First Australasian Conference, LNCS 1172, Springer-Verlag 1996, pp.79-86.

9. Xianmo Zhang, Yuliang Zheng. Cryptographically resilient functions. IEEE Tran. on Info. theory, 1997, Vol.43. No.5, PP.1740-1747.

10. Guozhen Xiao, J.Massey. A special characterization of correlation immune combining functions,IEEE Tran. on Info. theory,1988, Vol.34, PP.569-571.

11. Yixian Yan, Xuduan Lin. Coding theory and cryptography. People post and telecomunication publisher, 1992. (in chinese). 